\title{
Heterologous expression of the human ZIP4 zinc transporter in Saccharomyces cerevisiae
}

\author{
Yuting Liu, Elizabeth M. Bafaro, Robert E. Dempski*
}

Department of Chemistry and Biochemistry, Worcester Polytechnic Institute, Worcester, MA 01609, U.S.

To whom correspondence should be addressed: Robert E. Dempski, Department of Chemistry and Biochemistry, Worcester Polytechnic Institute, 100 Institute Road, Worcester, MA 01609, USA, Tel: (508) 831-4193; E-mail: rdempski@wpi.edu 


\section{ABSTRACT}

The human (h) transporter, hZIP4 is the primary zinc importer in the intestine and is also expressed in a variety of organs such as the pancreas and brain. Dysfunction of hZIP4 can result in the zinc deficiency disease acrodermatitis enteropathica (AE), which disrupts digestive and immune system homeostasis. Structure-function studies of hZIP4 have been greatly hindered by the absence of a robust heterologous expression system. Here, we report the heterologous expression of hZIP4 in Saccharomyces cerevisiae. Both a wild type and a mutant S. cerevisiae strain, in which the endogenous zinc transporters are deleted, were used to test the expression and localization of an hZIP4-GFP fusion protein. A full-length hZIP4-GFP and a truncated membrane domain only (mhZIP4-GFP) protein were successfully produced and targeted to the plasma membrane in yeast. 


\section{Introduction}

The transition metal zinc is an essential nutrient for human health. For example, zinc is a required catalytic and structural cofactor for zinc-dependent enzymes and transcription factors, and zinc is also functional in cell signaling processes ${ }^{1-3}$. Befitting its central importance to human health, zinc deficiency can lead to immune system dysfunction, growth retardation and neurological disorders ${ }^{4}$. While zinc deficiency is primarily caused by inadequate dietary intake of zinc ${ }^{5}$, genetic factors also influence human zinc levels. There are two classes of zinc transporters that regulate intracellular zinc levels: ZIP (Zrt-, Irt-like protein; solute-linked carrier SLC39 family) proteins act to increase cytosolic zinc levels and ZnT (zinc transporter; SLC30 family) act to decrease cytosolic zinc levels. The zinc-deficiency disease acrodermatitis enteropathica is an autosomal recessive genetic disorder that can lead to death if left untreated. The genetic basis for acrodermatitis enteropathica was identified as the human (h) ZIP4 gene $(S L C 39 A 4)^{6,7}$. hZIP4 is expressed in a variety of cell types including the intestine ${ }^{8}$, the primary location of zinc uptake, as well as the pancreas ${ }^{9}$. In addition, overexpression of hZIP4 has been shown to increase the expression of proteins that can initiate or progress pancreatic cancer ${ }^{10}$.

Our current understanding of the biochemical and structure-function basis of ZIP4 comes from studies using mice $^{7}$, cultured cells ${ }^{11}$ and Xenopus laevis oocytes ${ }^{12}$, as well as computational modeling ${ }^{13}$. Studies in mice showed that expression of mouse (m) ZIP4, which shares $75 \%$ sequence similarity to hZIP4, is regulated by dietary zinc levels ${ }^{8}$. Zinc-replete conditions stimulate mZIP4 endocytosis in cultured cells heterologously expressing $\mathrm{mZIP} 4^{14}$. Further, hZIP4 expressed in cultured cells undergoes ubiquitination and degradation at high intracellular zinc concentrations, and this requires a histidine-rich region on the largest intracellular loop of hZIP $4^{11}$. The large intracellular loop of hZIP4 is an intrinsically disordered protein region that binds two zinc ions with high affinity through the cysteine and histidine-rich region as shown by in vitro studies with the purified protein region ${ }^{15}$. In situ expression of hZIP4 in X. laevis oocytes has shown that this protein can also transport copper (II), nickel (II) and iron (II) ${ }^{12}$, and metal transport requires conserved histidines in the transmembrane region $^{13}$. The hZIP4 metal transport pathway has been inferred from structural models generated using Rosetta $a b$ initio modeling with co-evolution contact prediction ${ }^{13}$ and using homology modeling based on an X-ray crystal structure of a bacterial ZIP homolog ${ }^{16}$. Both models predicted a core of four transmembrane segments (TM2, 4, 5 and 7) with conserved charged residues (histidine, aspartate) lining the putative metal transport pathway. Direct structural information on hZIP4 is hampered by the insufficient quantity of purified protein attainable from the heterologous expression systems in cultured cells and $X$. laevis oocytes.

Saccharomyces cerevisiae is a robust, single cell eukaryotic model system that can be used to investigate the mechanism of heterologously expressed proteins, including transition metal transporters. In general, the advantages of $S$. cerevisiae include its fully sequenced genome, its genetic tractability using classical genetic approaches, and its ability to grow on chemically defined media ${ }^{17}$. As a model organism for transition metal 
expression, the genes involved in S. cerevisiae metal transport have been identified, many of which are homologous to metal transporter genes from other organisms including humans ${ }^{17}$. A variety of yeast transitionmetal transport deficient strains have been generated and used to characterize heterologously expressed metal transporters, including iron ${ }^{18}$, copper $^{19}$ and zinc ${ }^{20}$.

Since it is often difficult to produce large quantities of eukaryotic membrane proteins for high-resolution biochemical and structural studies, S. cerevisiae has been explored as a host organism to overexpress membrane proteins using GFP-based fusion technology. As the C-terminal GFP folds and becomes fluorescent only if the upstream membrane protein integrates into the membrane, the resultant fluorescence is a fast and accurate measure of membrane-integrated expression ${ }^{21}$. A reliable, high through-put protocol for overexpression and screening of eukaryotic membrane proteins in S. cerevisiae was developed ${ }^{22}$. Using GFP-fusion technology, membrane proteins can be quickly identified and screened via localization study. Confocal microscopy analysis of several eukaryotic membrane protein-GFP fusions under overexpression conditions demonstrated targeting of the overexpressed membrane proteins to the correct organelle in S. cerevisiae. Through the adjustment of the protein inducing conditions, maximal target protein levels can be obtained for further purification studies, and the membrane protein-GFP fusion can be purified by fluorescence size-exclusion chromatography ${ }^{23}$. To date, no human ZIP protein has been overexpressed in S. cerevisiae. In this study, we have successfully expressed hZIP4 tagged with green fluorescent protein (GFP) in S. cerevisiae and show that the hZIP4-GFP fusion protein is targeted to the yeast plasma membrane under optimal protein expression conditions

\section{Results}

\section{hZIP4 is heterologously expressed in S. cerevisiae and localizes to the plasma membrane.}

C-terminal GFP fusions were used as a reporter (Figure 1) to monitor the S. cerevisiae heterologous expression and localization of hZIP4 or the truncated mhZIP4, which is the hZIP4 membrane domain only (Figure 2). hZIP4-GFP (or mhZIP4-GFP) protein expression was induced by $2 \%(\mathrm{w} / \mathrm{v})$ galactose and the relative GFP levels were measured in wild type (DY1457) and ZHY3 (zinc transport deficient strain ZHY3, $\Delta z r t 1 / \Delta z r t 2$ ). In both wild type and ZHY3, hZIP4-GFP and mhZIP4-GFP were expressed as indicated by the increase in fluorescence compared to the background empty vector controls (Figure $3 \mathrm{~A}$ ). Expression levels in the zinc transporter deficient ZHY3 strain were twice as high as in the wild type DY1457 strain. Additionally, the truncated, membrane domain hZIP4-GFP fusion expression was two-fold higher than the full-length hZIP4-GFP, regardless of the strain.

The cellular localization of hZIP4-GFP and mhZIP4-GFP was assessed using confocal microscopy (Figure 3 B). As expected, the fluorescence levels were low for both yeast strains when transformed with the empty vector. In contrast, congruent with the total fluorescence levels observed (Figure $3 \mathrm{~A}$ ), an increase in fluorescence was 
observed for hZIP4-GFP and mhZIP4-GFP in both strains. Here, fluorescent rings on the outside of the cells indicate localization of the proteins to the plasma membrane of the yeast cells. In cells expressing higher protein levels, some fluorescent protein accumulated in subregions of the plasma membrane. This punctuated distribution pattern has been observed for other proteins that localize in microdomains within the plasma membrane ${ }^{24}$. Together, these results demonstrate that hZIP4-GFP and mhZIP4-GFP can be heterologously expressed on the surface of $S$. cerevisiae.

Role of hZIP4 in Growth Rate Control. To assess the role of hZIP4-GFP and mhZIP4-GFP expressed in S. cerevisiae, growth curves were measured with ZHY3 and wild type strains. Both strains were transformed with the yeast empty vector (EV), hZIP4-GFP or mhZIP4-GFP. Growth curves were obtained in liquid media under non-inducing (glucose) or inducing (galactose) conditions with high $(2 \mathrm{mM})$ or low $(0.5 \mathrm{mM})$ zinc (media was chelex-treated by adding $1 \mathrm{mM}$ EDTA). Under non-inducing condition, with high zinc levels, growth curves for ZHY3 or wild type transformed with the empty vector, hZIP4-GFP or mhZIP4-GFP plasmids were indistinguishable (Figure $4 \mathrm{~A}$ ). For all the cell types, the lag phases were identical, and, during exponential growth, the doubling times $\left(T_{D}\right)$ were less than two hours. When the same cells were grown under zinc limiting conditions, differences between the wild-type and zinc transport-deficient mutant ZHY3 strains were observed (Figure 4 B). In zinc deficient media, the growth curves of wild type strain transformed with the empty vector, hZIP4-GFP or mhZIP4-GFP plasmids were similar to those seen in zinc replete media. However, for the ZHY3 strain transformed with the empty vector, hZIP4-GFP or mhZIP4-GFP plasmids, the exponential growth rate in zinc deficient media was significantly slower compared to wild type strain (Figure $4 \mathrm{~B}$ ). This result was expected as the ZHY3 strain is missing both zinc importers and grows slowly in zinc deficient media ${ }^{25}$. Quantitatively, $T_{D}$ for each of the transformed wild type cells remained at two hours, whereas, $T_{D}$ for each of the transformed ZHY3 cells were more than six hours in zinc-limiting media.

In contrast to non-inducing protein conditions where growth curves were similar for ZHY3 or wild type whether or not they were transformed with hZIP4-GFP or mhZIP4-GFP, significant differences in growth curves were observed for both ZHY3 and wild type cells upon hZIP4 or mhZIP4 protein expression when compared to the cells transformed with the empty vector grown under inducing (galactose) conditions (Figure $4 \mathrm{C}-\mathrm{F}$ ). In the presence of high levels of zinc (Figure 4 D, F), growth curves for the ZHY3 and wild type expressed with empty vector were similar to those seen in non-inducing conditions. In contrast, induction of hZIP4 or mhZIP4-GFP for both strains resulted in cell growth that was significantly slower, and does not reach exponential phase, compared to the cells transformed with the empty vector. Expression of hZIP4-GFP in both wild type and ZHY3 resulted in an even slower lag phase than mhZIP4-GFP expressing cells in inducing conditions and in zinc replete media. In the presence of protein inducing conditions and zinc limited media $\left(0.5 \mathrm{mM} \mathrm{Z \textrm {n } ^ { 2 + }}\right)$, wild type cells transformed with the empty vector grew significantly faster than the ZHY3 strain (Figure 4 C, E). Again, it was observed that heterologous expression of mhZIP4-GFP resulted in a significantly slower growth rate for both 
wild type (DY1457)and ZHY3 cells when compared to the empty vector (Figure 4 C, E). In addition, heterologous expression of hZIP4-GFP resulted in a larger decrease in cell growth when compared to cells transformed with mhZIP4-GFP.

\section{Discussion}

hZIP4 is a plasma membrane protein expressed in various cell types including the intestine and pancreas ${ }^{8,9}$. Mutations in hZIP4 lead to a lethal genetic disorder acrodermatitis enteropathica ${ }^{26}$. Clearly, zinc homeostasis is essential for human health. Studies of ZIP4 expressed in mice, cultured cells and X. laevis oocytes have provided useful insights into the regulation of hZIP4, its metal substrate specificity and predicted metal transport pathway $^{8,14,12,13}$. S. cerevisiae provides an attractive alternative for heterologous expression of metal transporters because the metal homeostatic mechanisms are well-defined in yeast ${ }^{17}$. We report here the first yeast-based heterologous expression system for a mammalian ZIP protein. Two hZIP4-GFP fusions, one for the full-length transporter and one for the membrane domain only (mhZIP4), were expressed in wild-type (DY1457) and zinc transport-deficient (ZHY3) S. cerevisiae strains. GFP fluorescence levels and confocal microscopy confirmed the successful expression and plasma membrane localization of the hZIP4-GFP and mhZIP4-GFP fusion proteins.

The ZIP family of metal transporters has been shown to increase cytosolic zinc levels in vivo. Deletion of the two ZIP genes, zrt1 and zrt2, in S. cerevisiae, produces a growth phenotype sensitive to zinc-limited conditions. Thus, functional expression of hZIP4 in the zrt1/zrt2 deletion strain S. cerevisiae ZHY3 was expected to restore its zinc-dependent growth rate, as has been shown for a number of plant and fungal ZIP homologues that have been expressed in ZHY3 ${ }^{27-29}$. Surprisingly, growth of ZHY3 expressing either full-length hZIP4 or the hZIP4 membrane domain was repressed further in zinc-limited growth conditions. Similarly, growth of the wild-type strain S. cerevisiae DY1457, which has normal growth on both zinc-replete and zinc-deficient media, was repressed upon induction of hZIP4-GFP or mhZIP4-GFP protein expression. The repressed growth rates observed upon expression of hZIP4-GFP or mhZIP4-GFP could be due to an increased metabolic burden associated with heterologous membrane protein overexpression or toxicity of the expressed protein in yeast.

Another interesting result derived from our yeast-based hZIP4 expression system is that the truncated, membrane domain of hZIP4 was expressed and targeted to the yeast plasma membrane and also had the same effect on cell growth rate compared to the full-length hZIP4. The large N-terminal ectodomain is cleaved under extended zinc deficiency, and mutations in the N-terminus have been identified in acrodermatitis enteropathica cases $^{30}$. The study by Kambe and Andrews demonstrated that the processed mouse ZIP4, consisting of the membrane domain after ectodomain cleavage, was functional for zinc uptake in mouse and human cells ${ }^{30}$. However, a more recent study in HEK293T cells showed that the truncated hZIP4 was significantly impaired for zinc uptake ${ }^{31}$. Our heterologous yeast-based expression system is advantageous over mammalian cell lines, because the yeast 
system provides a well-defined metal transport background. In addition, the yeast-based system for heterologous membrane protein expression is easily monitored using GFP levels and confocal microscopy, since the Cterminal GFP folds and becomes fluorescent only if the upstream membrane protein integrates into the membrane. The yeast-based hZIP4 expression system developed here could be a valuable tool to express and purify the proteins for functional reconstitution studies or structural studies.

Among the advantages of using a yeast model system for membrane protein expression, recent studies have shown that $S$. cerevisiae is flexible in high-throughput fluorescent-based eukaryotic membrane protein overexpression $^{22}$, and yeast is considered to be a facile platform for high-throughput screening of protein inhibitors or activators ${ }^{32}$. Although yeast-based screening may not be as accurate as mammalian cells due to differences in post-translational modification and other physiological differences, yeast-based assays are more facile for high-throughput screening by simply monitoring cell growth. Currently, there are no known drugs that target to hZIP4 transporter, the yeast-based expression for hZIP4 described here may provide a useful tool for screening molecules that activate or inhibit the target hZIP4 protein.

\section{Methods}

Yeast strains, plasmids, media and reagents. The $S$. cerevisiae strains used in this study were kindly provided by the Eide Lab (University of Wisconsin-Madison): wild type strain DY1457 (MAT $\alpha$, ade6, can1,

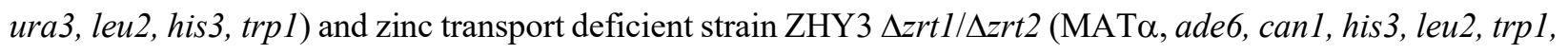
ura3, zrt1::LEU2, zrt2::HIS3). The plasmid pDDGFP-LEU2D was a gift from Simon Newstead (Addgene plasmid \# 58352). The plasmid pPICZ used to obtain the yeast $\alpha$-factor secretion signal sequence and was obtained from ThermoFisher Scientific. For the culture media, Yeast Peptone Dextrose (YPD) contained 1\% $(\mathrm{w} / \mathrm{v})$ yeast extract, $2 \%(\mathrm{w} / \mathrm{v})$ peptone and $2 \%(\mathrm{w} / \mathrm{v})$ glucose, which were purchased from Bacto ${ }^{\mathrm{TM}}$, HIMEDIA and Sigma, respectively. Synthetic Defined Culture (SDC) medium was made with $1.7 \%$ (w/v) zinc-free yeast nitrogen base (YNB-ZnSO 4$)$ (Sunrise Science Products), $5 \%$ (w/v) $\left(\mathrm{NH}_{4}\right)_{2} \mathrm{SO}_{4}(\mathrm{VWR})$, amino acid supplements without uracil (-URA) (US Biological Life Sciences), and 2\% (w/v) glucose (non-inducing) or galactose (inducing) (Sigma), then the media were adjusted to $\mathrm{pH} 4.2$ with $10 \mathrm{mM}$ citric-Na ${ }_{3}$ Citrate buffer (Fisher Scientific). Trace metal supplements, $\mathrm{MnCl}_{2}$ and $\mathrm{FeCl}_{3}$ were purchased from Alfa Aesar, and EDTA was obtained from Fisher Scientific. For confocal microscopy, Yeast Suspension Buffer (YSB) was used to slow the cells mobility, YSB was made of 5 mM EDTA, $50 \mathrm{mM}$ Tris- $\mathrm{HCl}$ pH7.6 and 10\% (v/v) glycerol.

Plasmid Construction. Two hZIP4 gene constructs were inserted into the yeast vector pDDGFP-LEU2D ${ }^{33}$ : the full length hZIP4 gene (residues 1-647) and a truncated construct (mhZIP4) encoding only the eight transmembrane domains (residues 328-647). The hZIP4 genes were preceded by the yeast $\alpha$-factor secretion signal sequence (Table 1). The genes were cloned into the pDDGFP-LEU2D vector, which encodes a C-terminal 
GFP, by homologous recombination in yeast cells that were transformed with the DNA insert and plasmid using the lithium acetate procedure ${ }^{34}$. Following insertion into the pDDGFP-LEU2D plasmid, the hZIP4 and mhZIP4 genes encoded a fusion protein with an N-terminal $\alpha$-factor secretion signal and a C-terminal GFP under the control of a gall promoter ${ }^{34}$. Yeast colonies were selected on SDC plates incubated at $30^{\circ} \mathrm{C}$ for 3 days. Plasmid sequences were confirmed by sequencing the entire gene.

S. cerevisiae hZIP4 Protein Expression and Localization. The expression of hZIP4 or mhZIP4 protein in S. cerevisiae wild type and zinc-deficient strains was assessed by quantifying the amount of GFP fluorescence after hZIP4 or mhZIP4 expression was induced by $2 \%(\mathrm{w} / \mathrm{v})$ galactose as described in Drew et al. $2008^{34}$. Yeast cells transformed with the empty vector were used as the control. After induction for 22-24 h, cells were harvested at $3000 \mathrm{~g}$ for $5 \mathrm{~min}$, washed and resuspended in YSB to an $\mathrm{OD}_{600}$ of 6. GFP fluorescence was measured using a PerkinElmer VICTOR ${ }^{3}$ Multilabel Counter using an excitation wavelength of $488 \mathrm{~nm}$ and an emission wavelength of $512 \mathrm{~nm}$ with the microplate set to bottom read ${ }^{34}$. For confocal microscopy, induced cells were resuspended in YSB, and a drop of cell culture was spotted on a 1\% agar pad (made with SDC medium) on a glass slide. The agar pad was sealed with VALAP (1:1:1 parts of vaseline, lanoline and paraffin) by cover slip ${ }^{35}$. Samples were focused with transmitted light at 10X magnification, then switched to blue light to estimate the gross localization of GFP. Laser scanning confocal microscopy was performed on a Leica TCS SP5 confocal microscope. Laser beams with $488 \mathrm{~nm}$ excitation wavelengths were used for FITC. Emission filter was 505 to 530 band pass for FITC. Single confocal sections and z-stack images were processed in Image $\mathrm{J}^{34,36}$.

Measurement of Growth Curves. Yeast cells were transformed, grown and harvested as above, then switched to $2 \%(\mathrm{w} / \mathrm{v})$ glucose or $2 \%(\mathrm{w} / \mathrm{v})$ galactose SDC for measuring the growth under non-inducing condition or inducing condition, respectively. Two zinc concentrations ( $2 \mathrm{mM}$ and $0.5 \mathrm{mM})$ were applied to each condition. The liquid culture was incubated at $30^{\circ} \mathrm{C}$ with $220 \mathrm{rpm}$ shaking for $30 \mathrm{~h}$ or $120 \mathrm{~h}$ starting at an initial $\mathrm{OD}_{600}$ of 0.1 . Cell growth was monitored by measuring $\mathrm{OD}_{600}$ as a function of time.

\section{Acknowledgments}

This work was supported by the NIH (R01 GM105964) to R.E.D. We thank Dr. Eide (University of WisconsinMadison) for providing the S. cerevisiae wild type strain DY1457 and zinc transport deficient strain ZHY3 $\Delta z r t 1 / \Delta z r t 2$.

\section{Author Contributions}

Y.L, E.M.B and R.E.D. designed the research; Y.L. performed the research and R.E.D. supervised the project.

\section{Additional Information}

Competing financial interests: The authors declare no competing financial interests. 


\section{References}

[1] Jeong, J., and Eide, D. J. (2013) The SLC39 family of zinc transporters, Molecular Aspects of Medicine 34, 612-619.

[2] Beyersmann, D., and Haase, H. (2001) Functions of zinc in signaling, proliferation and differentiation of mammalian cells, Biometals 14, 331-341.

[3] Bafaro, E., Liu, Y., Xu, Y., and Dempski, R. E. (2017) The emerging role of zinc transporters in cellular homeostasis and cancer, Signal transduction and targeted therapy 2, 17029.

[4] Prasad, A. S. (2013) Discovery of Human Zinc Deficiency: Its Impact on Human Health and Disease, Advances in Nutrition 4, 176-190.

[5] Roohani, N., Hurrell, R., Kelishadi, R., and Schulin, R. (2013) Zinc and its importance for human health: An integrative review, Journal of research in medical sciences : the official journal of Isfahan University of Medical Sciences 18, 144-157.

[6] Wang, K., Zhou, B., Kuo, Y.-M., Zemansky, J., and Gitschier, J. (2002) A Novel Member of a Zinc Transporter Family Is Defective in Acrodermatitis Enteropathica, The American Journal of Human Genetics 71, 66-73.

[7] Küry, S., Dréno, B., Bézieau, S., Giraudet, S., Kharfi, M., Kamoun, R., and Moisan, J.-P. (2002) Identification of SLC39A4, a gene involved in acrodermatitis enteropathica, Nature genetics 31, 239.

[8] Dufner-Beattie, J., Wang, F., Kuo, Y.-M., Gitschier, J., Eide, D., and Andrews, G. K. (2003) The Acrodermatitis Enteropathica Gene ZIP4 Encodes a Tissue-specific, Zinc-regulated Zinc Transporter in Mice, Journal of Biological Chemistry 278, 33474-33481.

[9] Li, M., Zhang, Y., Liu, Z., Bharadwaj, U., Wang, H., Wang, X., Zhang, S., Liuzzi, J. P., Chang, S.M., Cousins, R. J., Fisher, W. E., Brunicardi, F. C., Logsdon, C. D., Chen, C., and Yao, Q. (2007) Aberrant expression of zinc transporter ZIP4 (SLC39A4) significantly contributes to human pancreatic cancer pathogenesis and progression, Proceedings of the National Academy of Sciences 104, 18636.

[10] Zhang, Y., Bharadwaj, U., Logsdon, C. D., Chen, C., Yao, Q., and Li, M. (2010) ZIP4 Regulates Pancreatic Cancer Cell Growth by Activating IL-6/STAT3 Pathway via Zinc Finger Transcription Factor CREB, Clinical cancer research : an official journal of the American Association for Cancer Research 16, 1423-1430.

[11] Mao, X., Kim, B.-E., Wang, F., Eide, D. J., and Petris, M. J. (2007) A Histidine-rich Cluster Mediates the Ubiquitination and Degradation of the Human Zinc Transporter, hZIP4, and Protects against Zinc Cytotoxicity, Journal of Biological Chemistry 282, 6992-7000.

[12] Antala, S., and Dempski, R. E. (2012) The Human ZIP4 Transporter Has Two Distinct Binding Affinities and Mediates Transport of Multiple Transition Metals, Biochemistry 51, 963-973.

[13] Antala, S., Ovchinnikov, S., Kamisetty, H., Baker, D., and Dempski, R. E. (2015) Computation and Functional Studies Provide a Model for the Structure of the Zinc Transporter hZIP4, The Journal of Biological Chemistry 290, 17796-17805.

[14] Kim, B.-E., Wang, F., Dufner-Beattie, J., Andrews, G. K., Eide, D. J., and Petris, M. J. (2004) Zn2+-stimulated Endocytosis of the mZIP4 Zinc Transporter Regulates Its Location at the Plasma Membrane, Journal of Biological Chemistry 279, 4523-4530. 
[15] Bafaro, E. M., Antala, S., Nguyen, T.-V., Dzul, S. P., Doyon, B., Stemmler, T. L., and Dempski, R. E. (2015) The large intracellular loop of hZIP4 is an intrinsically disordered zinc binding domain, Metallomics : integrated biometal science 7, 1319-1330.

[16] Zhang, T., Liu, J., Fellner, M., Zhang, C., Sui, D., and Hu, J. (2017) Crystal structures of a ZIP zinc transporter reveal a binuclear metal center in the transport pathway, Science Advances 3, e1700344.

[17] Bleackley, M. R., and MacGillivray, R. T. A. (2011) Transition metal homeostasis: from yeast to human disease, BioMetals 24, 785-809.

[18] Dix, D. R., Bridgham, J. T., Broderius, M. A., Byersdorfer, C. A., and Eide, D. J. (1994) The FET4 gene encodes the low affinity $\mathrm{Fe}(\mathrm{II})$ transport protein of Saccharomyces cerevisiae, Journal of Biological Chemistry 269, 26092-26099.

[19] Wu, X., Sinani, D., Kim, H., and Lee, J. (2009) Copper transport activity of yeast Ctr1 is downregulated via its $\mathrm{C}$ terminus in response to excess copper, The Journal of biological chemistry 284, 4112-4122.

[20] Zhao, H., and Eide, D. (1996) The ZRT2 Gene Encodes the Low Affinity Zinc Transporter in Saccharomyces cerevisiae, Journal of Biological Chemistry 271, 23203-23210.

[21] Drew, D. E., von Heijne G Fau - Nordlund, P., Nordlund P Fau - de Gier, J. W., and de Gier, J. W. Green fluorescent protein as an indicator to monitor membrane protein overexpression in Escherichia coli.

[22] Newstead, S., Kim, H., von Heijne, G., Iwata, S., and Drew, D. (2007) High-throughput fluorescent-based optimization of eukaryotic membrane protein overexpression and purification in Saccharomyces cerevisiae, Proceedings of the National Academy of Sciences of the United States of America 104, 13936-13941.

[23] Kawate, T., and Gouaux, E. Fluorescence-detection size-exclusion chromatography for precrystallization screening of integral membrane proteins.

[24] Malínská, K., Malínský, J., Opekarová, M., and Tanner, W. (2003) Visualization of protein compartmentation within the plasma membrane of living yeast cells, Molecular biology of the cell 14, 4427-4436.

[25] Zhao, H., and Eide, D. J. (1997) Zap1p, a metalloregulatory protein involved in zinc-responsive transcriptional regulation in Saccharomyces cerevisiae, Molecular and Cellular Biology 17, 5044.

[26] Johnson, P., Federico, M., Kirkwood, A., Fosså, A., Berkahn, L., Carella, A., d'Amore, F., Enblad, G., Franceschetto, A., Fulham, M., Luminari, S., O’Doherty, M., Patrick, P., Roberts, T., Sidra, G., Stevens, L., Smith, P., Trotman, J., Viney, Z., Radford, J., and Barrington, S. (2016) Adapted Treatment Guided by Interim PET-CT Scan in Advanced Hodgkin's Lymphoma, New England Journal of Medicine 374, 2419-2429.

[27] Lee, J.-H., You, J., Dobrota, E., and Skalnik, D. G. (2010) Identification and Characterization of a Novel Human PP1 Phosphatase Complex, Journal of Biological Chemistry 285, 2446624476.

[28] Kozak, K., Papierniak, A., Barabasz, A., Kendziorek, M., Palusińska, M., Williams, L. E., and Antosiewicz, D. M. (2019) NtZIP11, a new Zn transporter specifically upregulated in tobacco leaves by toxic Zn level, Environmental and Experimental Botany 157, 69-78.

[29] Khouja, H. R., Abbà, S., Lacercat-Didier, L., Daghino, S., Doillon, D., Richaud, P., Martino, E., Vallino, M., Perotto, S., Chalot, M., and Blaudez, D. (2013) OmZnT1 and OmFET, two metal 
transporters from the metal-tolerant strain $\mathrm{Zn}$ of the ericoid mycorrhizal fungus Oidiodendron maius, confer zinc tolerance in yeast, Fungal Genetics and Biology 52, 5364.

[30] Kambe, T., and Andrews, G. K. (2009) Novel Proteolytic Processing of the Ectodomain of the Zinc Transporter ZIP4 (SLC39A4) during Zinc Deficiency Is Inhibited by Acrodermatitis Enteropathica Mutations, Molecular and Cellular Biology 29, 129.

[31] Zhang, T., Sui, D., and Hu, J. (2016) Structural insights of ZIP4 extracellular domain critical for optimal zinc transport, Nature Communications 7, 11979.

[32] Kawada, H., Inanobe, A., and Kurachi, Y. (2016) Isolation of proflavine as a blocker of G protein-gated inward rectifier potassium channels by a cell growth-based screening system, Neuropharmacology 109, 18-28.

[33] Parker, J. L., and Newstead, S. (2014) Method to increase the yield of eukaryotic membrane protein expression in Saccharomyces cerevisiae for structural and functional studies, Protein Science 23, 1309-1314.

[34] Drew, D., Newstead, S., Sonoda, Y., Kim, H., von Heijne, G., and Iwata, S. (2008) GFP-based optimization scheme for the overexpression and purification of eukaryotic membrane proteins in Saccharomyces cerevisiae, Nature Protocols 3, 784.

[35] Vidali, L., Rounds, C. M., Hepler, P. K., and Bezanilla, M. (2009) Lifeact-mEGFP reveals a dynamic apical F-actin network in tip growing plant cells, PloS one 4, e5744-e5744.

[36] Hermida-Matsumoto, L., and Resh, M. D. (2000) Localization of human immunodeficiency virus type $1 \mathrm{Gag}$ and Env at the plasma membrane by confocal imaging, Journal of virology $74,8670-8679$.

Table 1. Primer sequences. Full length and truncated hZIP4 primer sequences. Primers of hZIP4 and mhZIP4 S. cerevisiae signal sequence.

Figure 1. C-terminal fusion GFP hZIP4 and mhZIP4 plasmid. hZIP4 or mhZIP4 was amplified and inserted into pDDGFP-LEU2D vector with $\mathrm{C}$-terminal fusion GFP as indicator. The expression of hZIP4 or mhZIP4 was induced by galactose.

Figure 2. plasmid maps of hZIP4 and mhZIP4. The full length of hZIP4 and mhZIP4 was cloned into pDDGFP-LEU2D vector with GFP at C-terminal. GAL1 promoter allows protein expression induced by galactose.

Figure 3. hZIP4 and mhZIP4 expression in Saccharomyces cerevisiae wild type strain (WT) and zinc transport deficient strain (ZHY3). (a) Protein expression is indicated by the normalized fluorescence in arbitrary units (a.u.) of the reporter GFP. The background fluorescence was measured using cells transformed with the yeast empty vector (EV) pDDGFP-LEU2D. Data represent means \pm SD $(n=9)$. (b) Cellular localization of hZIP4 and mhZIP4 expressed in WT and 
ZHY3. All the transformed cells were induced with galactose before visualization by confocal microscopy. Cells transformed with the empty vector (EV) are shown as control.

Figure 4. Growth curve of ZHY3 and WT transformed with hZIP4 and mhZIP4. All cells were cultured in synthetic defined culture (SDC) with glucose overnight. Cells were diluted to OD600 0.1 in SDC with glucose (non-inducing) or galactose (inducing) under zinc-deficient $(0.5$ $\mathrm{mM})$ and zinc-replete $(2 \mathrm{mM})$ conditions. $(\mathrm{a}, \mathrm{b}) \mathrm{ZHY} 3$ and WT with hZIP4, mhZIP4 and EV in glucose. (c,d) ZHY3 with hZIP4, mhZIP4 and EV in galactose. (e,f) WT with hZIP4, mhZIP4 and $\mathrm{EV}$ in galactose. 


\begin{tabular}{|c|c|}
\hline Sequences & Primer sequence (5'-3') \\
\hline hZIP4 S. cere signal sequence & ACCCCGGATTCTAGAACTAGTGGATCCCCCATGAGATTTCCTTCA \\
\hline forward & ATTTTTACTGC \\
\hline $\begin{array}{l}\text { hZIP4 full-length overlap } \\
\text { extension forward }\end{array}$ & GAGAGGCTGAAGCTTACGTAGCGTCCCTGGTCTCGCTGGAGC \\
\hline $\begin{array}{l}\text { hZIP4 full-length overlap } \\
\text { extension reverse }\end{array}$ & GCTCCAGCGAGACCAGGGACGCTACGTAAGCTTCAGCCTCTC \\
\hline hZIP4 S. cere signal sequence & AAATTGACCTTGAAAATATAAATTTTCCCCAGAACCACCGAAGGT \\
\hline reverse & GATGTCATCCTCGTAC \\
\hline $\begin{array}{l}\text { truncated hZIP4 } S \text {. cere signal } \\
\text { sequence forward }\end{array}$ & $\begin{array}{l}\text { ACCCCGGATTCTAGAACTAGTGGATCCCCCATGAGATTTCCTTCA } \\
\text { ATTTTTACTGC }\end{array}$ \\
\hline $\begin{array}{l}\text { truncated hZIP4 full-length } \\
\text { overlap extension forward }\end{array}$ & GAGAGGCTGAAGCTTACGTACTGTACGGCTCCCTGGCCACGC \\
\hline $\begin{array}{l}\text { truncated hZIP4 full-length } \\
\text { overlap extension reverse }\end{array}$ & GCGTGGCCAGGGAGCCGTACAGTACGTAAGCTTCAGCCTCTC \\
\hline $\begin{array}{l}\text { truncated hZIP4 S. cere signal } \\
\text { sequence reverse }\end{array}$ & $\begin{array}{l}\text { AAATTGACCTTGAAAATATAAATTTTCCCCAGAACCACCGAAGGT } \\
\text { GATGTCATCCTCGTAC }\end{array}$ \\
\hline
\end{tabular}

Table 1. 
bioRxiv preprint doi: https://doi. org/10.1101/2021.09.27.461959. this version posted September 27, 2021. The copyright holder for this preprint (which was not certified by peer review) is the author/funder, who has granted bioRxiv a license to display the preprint in perpetuity. It is made available under aCC-BY-NC-ND 4.0 International license.

TOP

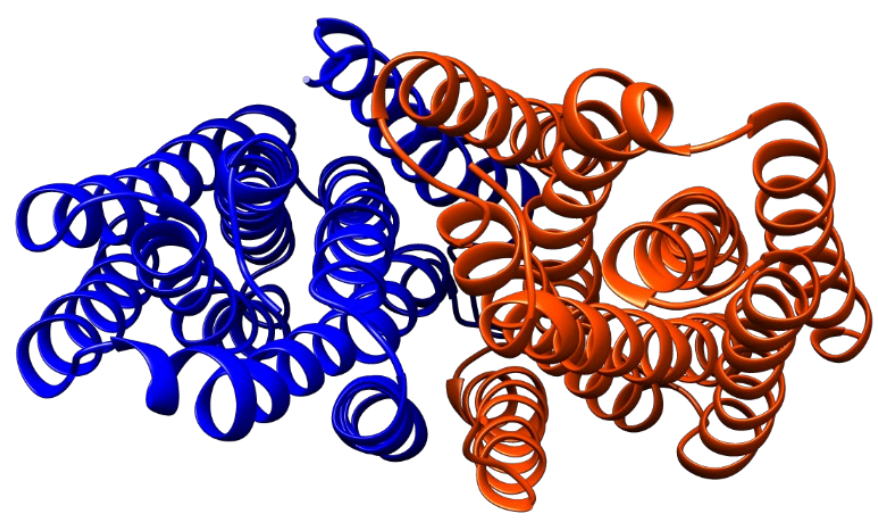

SIDE

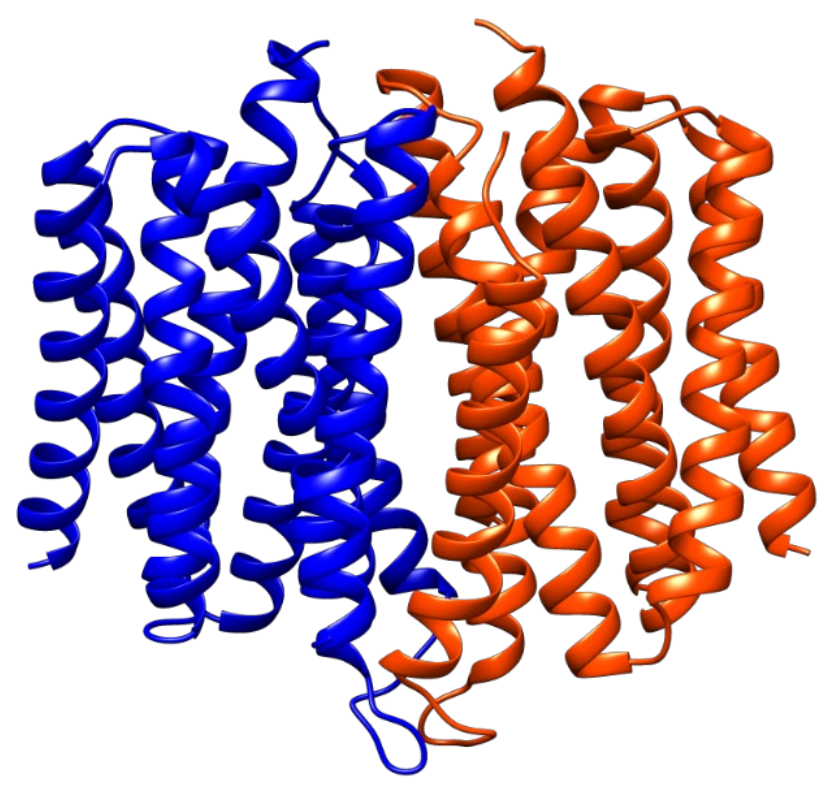

Figure 1. 
bioRxiv preprint doi: https://doi.org/10.1101/2021.09.27.461959; this version posted September 27, 2021. The copyright holder for this preprint (which was not certified by peer review) is the author/funder, who has granted bioRxiv a license to display the preprint in perpetuity. It is made available under aCC-BY-NC-ND 4.0 International license.

Forward primer

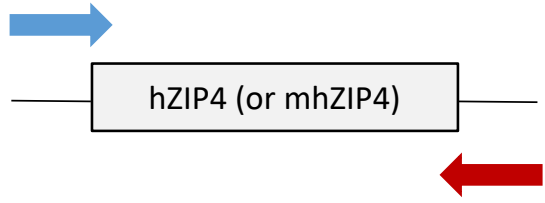

Reverse primer
Amplify hZIP4 (mhZIP4)
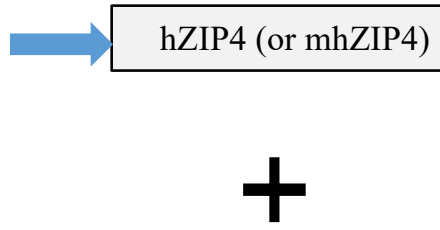

GAL 1 promoter
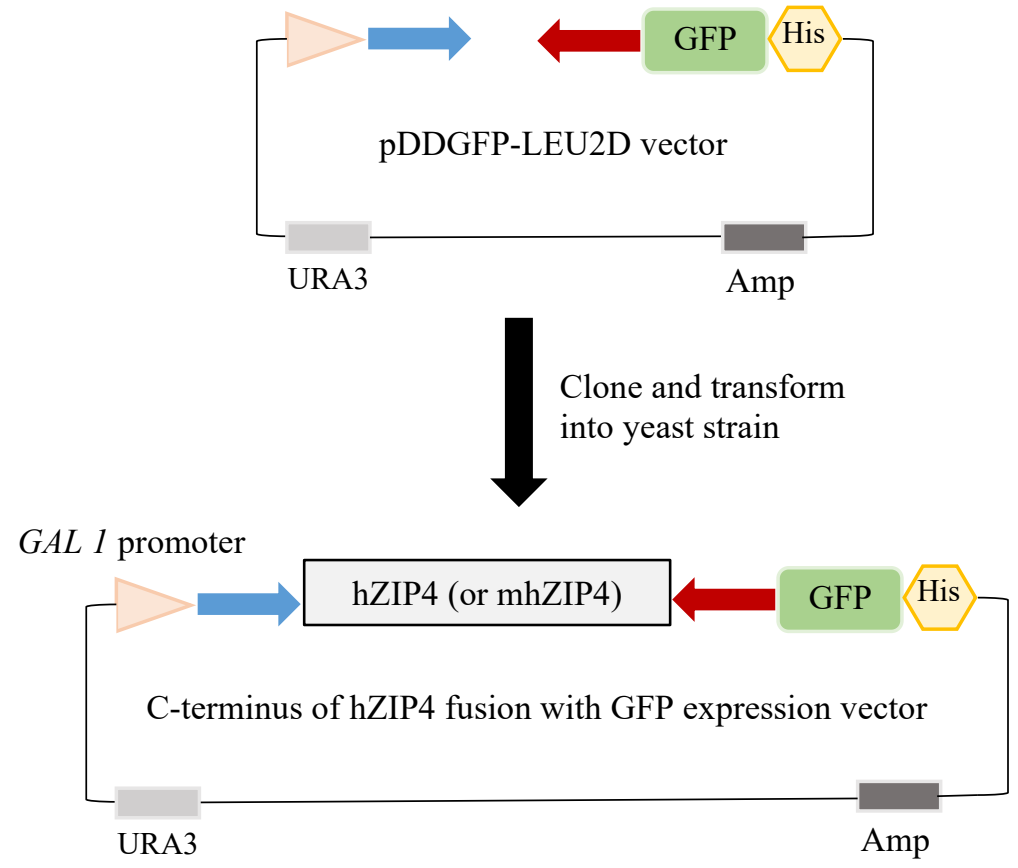

Figure 2. 
bioRxiv preprint doi: https://doi.org/10.1101/2021.09.27.461959; this version posted September 27, 2021. The copyright holder for this preprint (which was not certified by peer review) is the author/funder, who has granted bioRxiv a license to display the preprint in perpetuity. It is made available under aCC-BY-NC-ND 4.0 International license.
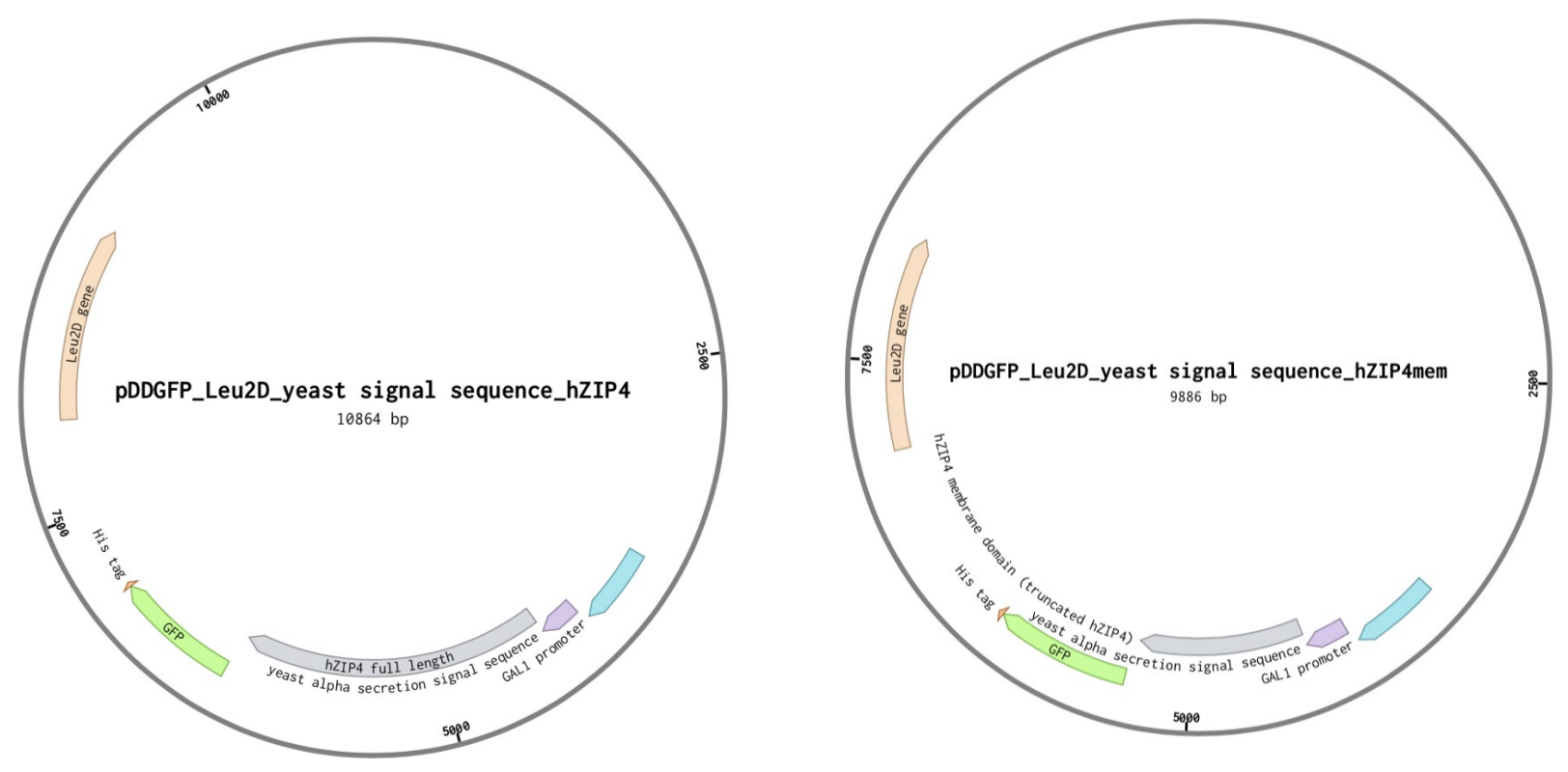

Figure 3. 
a

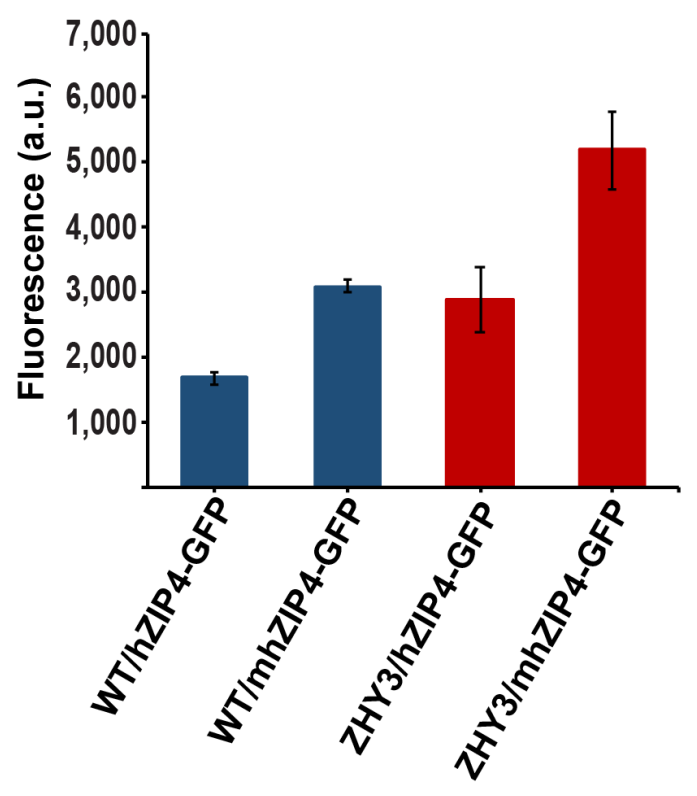

b
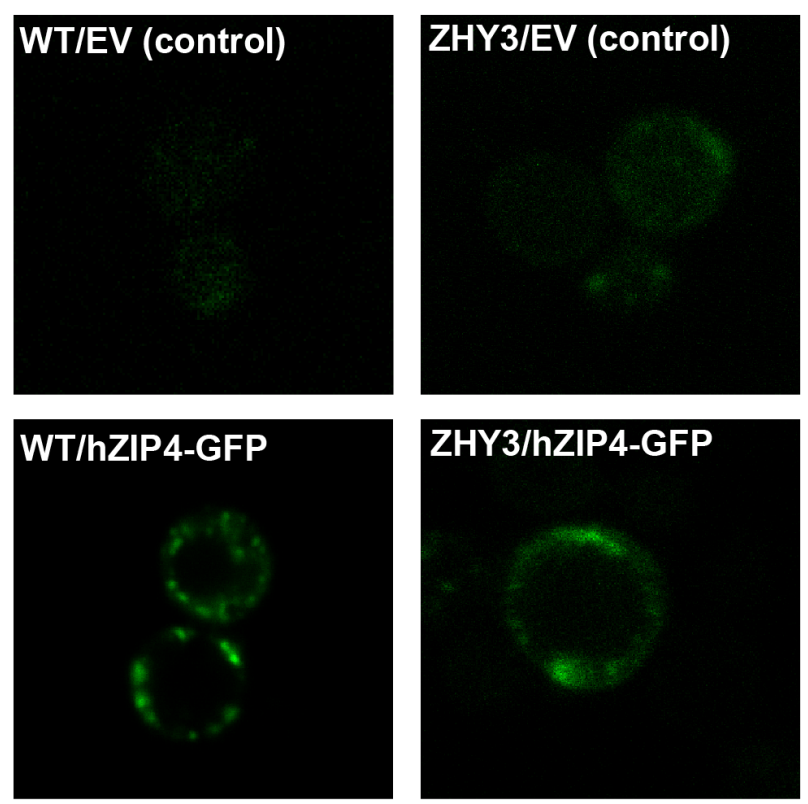

\section{ZHY3/hZIP4-GFP}
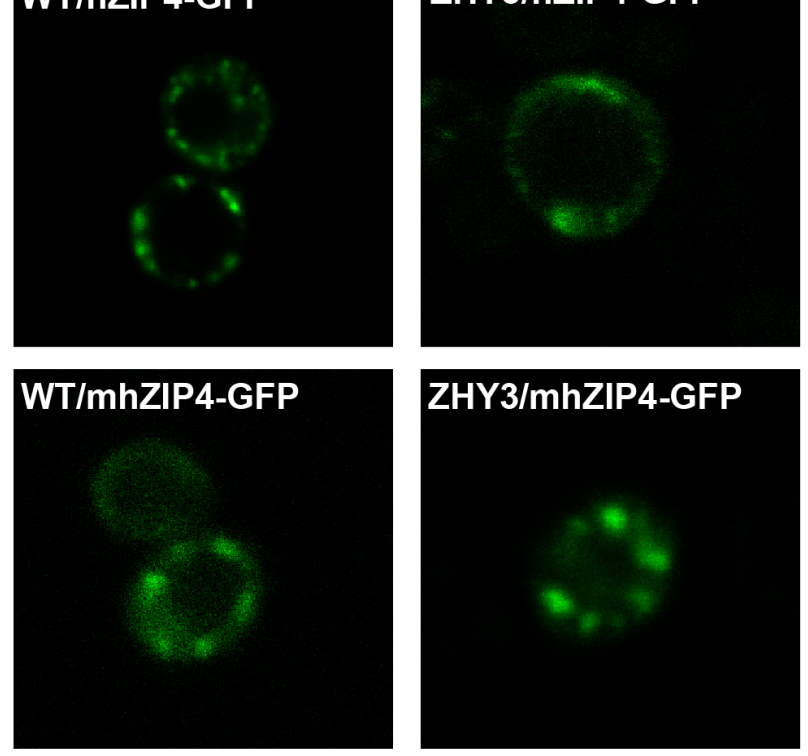

Figure 4. 
a

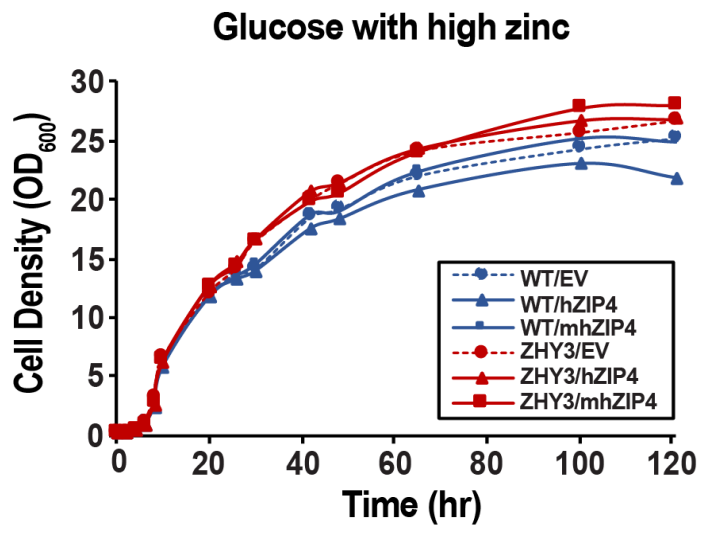

C

Galactose with low zinc (ZHY3)

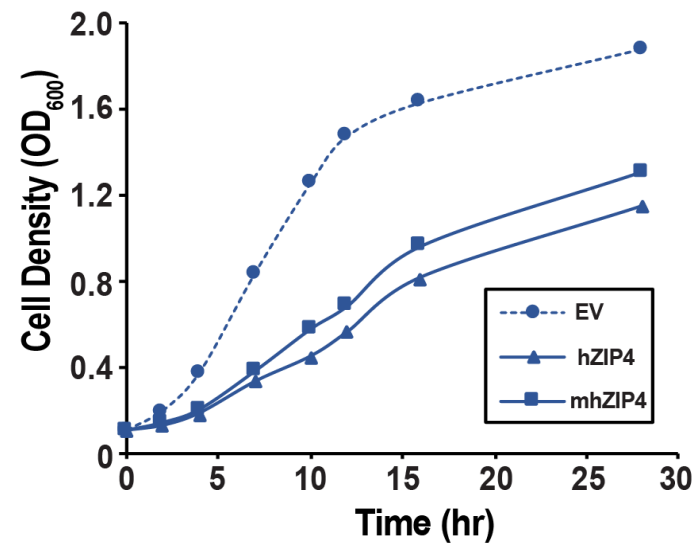

e

Galactose with low zinc (WT)

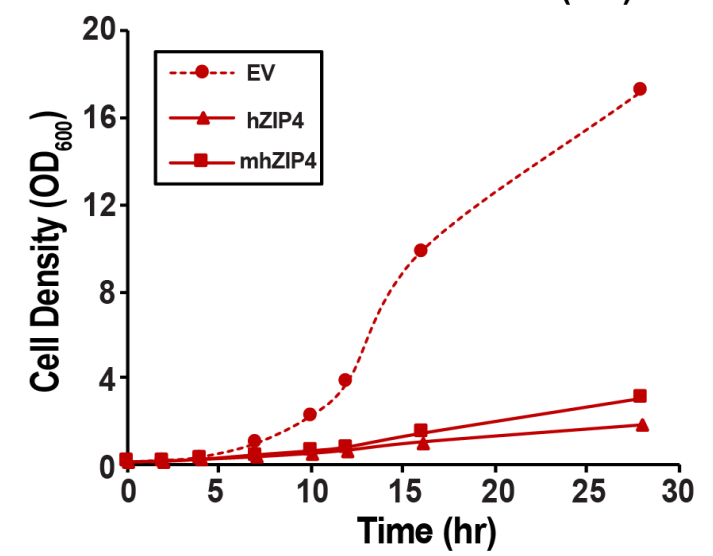

b

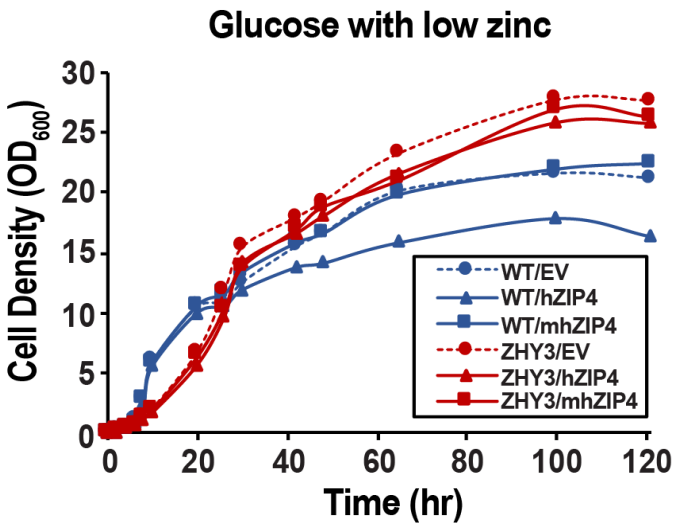

d

Galactose with high zinc (ZHY3)

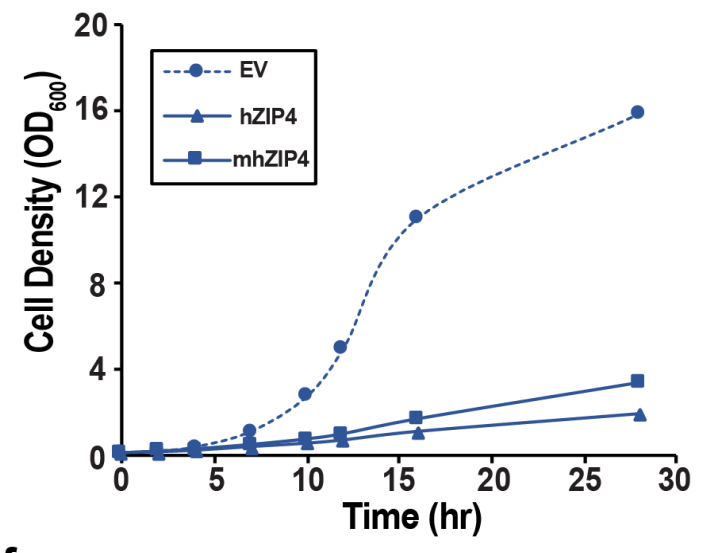

f

\section{Galactose with high zinc (WT)}

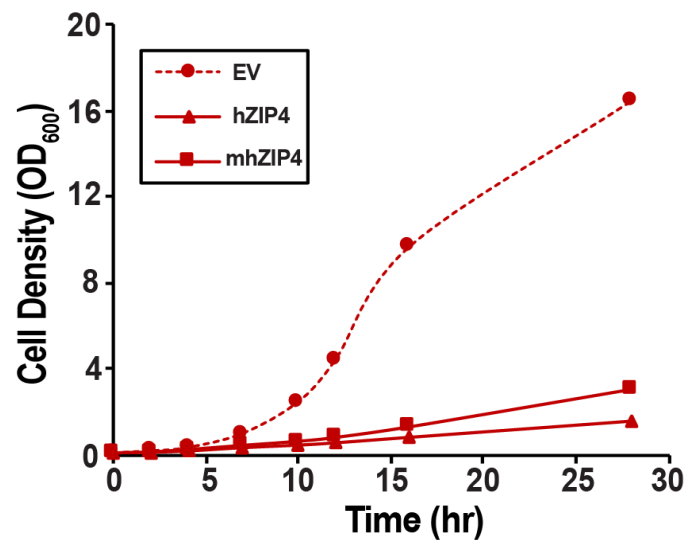

Figure 5. 